\title{
Review
}

Jonathan Shannahan*

\section{The biocorona: a challenge for the biomedical application of nanoparticles}

DOI 10.1515/ntrev-2016-0098

Received November 17, 2016; accepted December 8, 2016; previously published online January 20, 2017

\begin{abstract}
Formation of the biocorona on the surface of nanoparticles is a significant obstacle for the development of safe and effective nanotechnologies, especially for nanoparticles with biomedical applications. Following introduction into a biological environment, nanoparticles are rapidly coated with biomolecules resulting in formation of the nanoparticle-biocorona. The addition of these biomolecules alters the nanoparticle's physicochemical characteristics, functionality, biodistribution, and toxicity. To synthesize effective nanotherapeutics and to more fully understand possible toxicity following human exposures, it is necessary to elucidate these interactions between the nanoparticle and the biological media resulting in biocorona formation. A thorough understanding of the mechanisms by which the addition of the biocorona governs nanoparticle-cell interactions is also required. Through elucidating the formation and the biological impact of the biocorona, the field of nanotechnology can reach its full potential. This understanding of the biocorona will ultimately allow for more effective laboratory screening of nanoparticles and enhanced biomedical applications. The importance of the nanoparticle-biocorona has been appreciated for a decade; however, there remain numerous future directions for research which are necessary for study. This perspectives article will summarize the unique challenges presented by the nanoparticle-biocorona and avenues of future needed investigation.
\end{abstract}

Keywords: in vitro toxicology; nanotherapeutics; nanotoxicology; protein corona.

*Corresponding author: Jonathan Shannahan, School of Health Sciences, Purdue University, 550 Stadium Mall Dr. 47907, West Lafayette, Indiana, USA, Tel.: +765-494-2326,

e-mail: jshannah@purdue.edu

\section{Nanoparticle-biocorona overview}

Presentation of nanoparticles into a physiological environment results in the absorption of a variety of biological materials onto the surface of nanoparticles. These biological materials include proteins, peptides, lipids, nucleic acids, metabolites, and others. The association of these biomolecules is dependent on a number of factors including the physicochemical properties of the nanoparticle, the physiological environment, and the duration of incubation [1, 2]. This collection of biomolecules on the nanoparticle's surface is collectively referred to as the biocorona. The biocorona can be subdivided into portions: the first or primary layer termed as the hard biocorona and the secondary layers referred to as the soft biocorona. The hard biocorona is governed by nanoparticle-biomolecule interactions resulting in tightly associated biomolecules. These nanoparticle-biomolecular interactions are often driven by electrostatic interactions, hydrophobicity, and favorable entropyrelated shifts in biomolecule conformation [1, 3-6]. The soft biocorona, however, is more dynamic in nature than the hard biocorona due to the rapid exchange of biomolecules based on biomolecule-biomolecule interactions. Overall, the addition of the biocorona has been found to alter nanoparticle physicochemical characteristics, functionality, biodistribution, and toxicity [6-9]. For the development of safe and effective nanoparticles for biomedical applications, it is necessary for the biocorona to be understood. Investigation of the nanoparticlebiocorona is currently an important and impactful area of research; therefore, numerous thorough reviews have recently been published [1,10-13]. This perspectives article, however, aims to highlight and summarize the unique challenges involved in the study of the nanoparticle-biocoronaand to emphasize novel avenues of future research. 


\section{Challenges presented by the nanoparticle-biocorona}

\subsection{Evaluation of biocorona components}

Recent advances in the field of proteomics have driven the evaluation of the protein components forming the nanoparticle-biocorona [14, 15]. These studies are normally performed by incubating nanoparticles in a physiological medium (i.e. serum, plasma, or airway lining fluid) and allowing biomolecules to associate with the nanoparticle's surface. The nanoparticles with their newly added biocorona can then be separated from unassociated biomolecules typically via centrifugation and a series of washes. Associated proteins are then eluted from the surface of the nanoparticles and separated from the nanoparticles using either centrifugation or gel electrophoresis. Following reduction and digestion of the proteins, mass spectrometry and bioinformatics are utilized to identify and quantify these protein components of the biocorona. Studies have demonstrated that hundreds of proteins associate with the surface of the nanoparticle forming the biocorona in serum, with the most abundant protein components typically being serum albumin, apolipoproteins, complement system proteins, immunoglobulins, and $\alpha-2$ macrogobulin $[1,5,8,16]$. Although shared between nanoparticles, these proteins are often associated in different quantities based on the type of nanoparticle. Specifically, silver nanoparticles with a larger diameter demonstrated more abundance of these shared proteins when compared to smaller diameter silver nanoparticles [5]. Differences in surface coatings, however, were not found to influence abundance. This difference in quantity was hypothesized to be related to the differential surface curvature between the silver nanoparticles. Further, unique proteins are often present in the biocorona due to variations in nanoparticle properties. Many proteins are often also found to be enriched within the nanoparticle-biocorona compared to their quantity in the biological media. All nanoparticles do not associate the same proteins and therefore do not form identical biocoronas.

To more effectively screen the biological impact of the nanoparticle-biocorona, studies have evaluated simplified biocoronas consisting of only primary protein components. Production of a simplified biocorona on the surface of nanoparticles using selected highly abundant proteins, however, does not appear to fully simulate biological responses. The minor components appear to play a role in the biological responses to the nanoparticle-biocorona, demonstrating that these responses are not simply driven by the abundant proteins. Shannahan et al. investigated differences in cellular responses following exposure to silver nanoparticles with only a serum albumin biocorona or a complex biocorona following incubation in fetal bovine serum [17]. Cellular association of nanoparticles and inflammatory activation were not determined to be purely driven by bovine serum albumin even though it was the primary biocoronal protein. Further, the greater number of biocoronal proteins used in a multivariate model to predict nanoparticle-cell association was found to increase its accuracy compared to a model that only utilized individual proteins or only nanoparticle properties [18]. Walkey et al. demonstrated that 64 individual proteins were needed for a reliable model that was $86 \%$ more accurate than using nanoparticle characteristics without a corona [18]. Using 32 individual proteins only decreased model accuracy by $5 \%$ in comparison to the model using 64 proteins. These studies demonstrate how an individual protein or highly abundant proteins in the biocorona do not completely govern cell interactions with the activity of the nanoparticle-biocorona. Overall, this implies that individual protein biocoronas cannot be used as a surrogate for complete biocoronas, as a simplified biocorona is not entirely predictive of the biological activity of the complex biocorona.

Due to the unique biological identity imparted to the nanoparticle by addition of the biocorona, it is important to understand the spatial orientations of the proteins on the nanoparticle surface and what portions of the protein remain available for biological interactions. Recently, Kelly et al. demonstrated that transferrin-coated polystyrene nanoparticles randomly associated proteins in terms of spatial organization [19]. Further, this assessment found that only a few of the bound proteins presented external functional motifs suggesting that the proteins likely do not retain their functional capacity when bound [19]. This evaluation of the spatial organization of the biocoronal protein components as well as the presentation of functional motifs allows for more detailed examination of nanoparticle-biocorona to take place. Now that the tools for this type of analysis have been created, many avenues of future research are possible such as examining spatial and conformational changes of proteins on a variety of nanoparticles and using these data to predict cellular interactions and biological responses.

To date, evaluation of other non-protein biocoronal components, such as lipids and nucleic acids, is lacking. These lipid components may be significant in modifying the activity of the nanoparticle as they may stimulate uptake and/or activation of cells that normally remove lipids from circulation such as macrophages and endothelial cells. 
Recent study has demonstrated that incorporation of cholesterol into media during nanoparticle exposure resulted in increased macrophage uptake of nanoparticles possibly though adsorption of cholesterol onto the nanoparticle's surface [20]. It is likely that lipid-rich biocoronas may alter cellular interactions and responses through scavenger receptors expressed on the surface of a variety of cells including macrophages, endothelial cells, and others. Scavenger receptors are a protein supergroup known to specifically be involved in the uptake of negatively charged particles and oxidized lipids [21, 22]. All scavenger receptor classes have mammalian orthologues except for class C (only found in insects) [23]. This conservation of scavenger receptors points to their important biological role which may be taken advantage of for therapeutic benefit. Nanoparticles with lipid-rich biocoronas may directly target cells that express high levels of scavenger receptors on their surface. Through manipulating the length of polyethylene glycol chains on nanoparticles, researchers were able to alter the amount of apolipoprotein that associated with the biocorona [24]. These nanoparticles with apolipoprotein-rich biocoronas were found to more selectively bind scavenger receptors and increased uptake by cells in vitro [24]. Interactions between cell surface receptors and the biocorona on nanoparticles needs to be further evaluated as the implications of these interactions will relate to the nanoparticle's biopersistence, biodistribution, and cellular responses. It is likely that different biocoronas will target distinctive cell surface receptors resulting in differential responses. Due to the limited data that exist regarding non-protein components of the nanoparticlebiocorona, future studies will be needed to identify these components and the interactions that result in their association with the nanoparticle's surface. Specifically, to perform these studies, advancements in lipidomics and imaging technologies are needed.

As stated previously, the formation of the nanoparticle-biocorona is driven by the nanoparticle physicochemical properties, the physiological environment, and the incubation time. Numerous studies have evaluated the influence of nanoparticle physicochemical properties on biocorona formation to determine correlations between nanoparticle properties and specific biomolecule association $[18,25]$. Due to the interdependence of nanoparticle physicochemical properties as well as the diverse nature of nanoparticles, it is difficult to determine exact correlations between nanoparticle properties and biocoronal components [26]. In order to correlate the components of the biocorona with the unique nanoparticle physicochemical properties, it may be necessary to modify how correlations are performed. Instead of attempting to correlate individual nanoparticle properties (size, surface area, charge, shape, composition, and coating) and macromolecule properties (type, class, or size) it may be more beneficial in the future to examine correlations in new terms or combinations of properties. These could include new parameters and/or combinations of parameters such as surface curvature, electrostatic charge per surface area, conformationally available amino acids, interactive amino acid content, and others. Large, well-controlled study designs and the input of computer modelers are needed to systematically determine relationships between nanoparticle properties and components of the biocorona.

Although the hard corona is rather stable, it is possible for the biomolecule content to change over time. It is necessary, therefore, to evaluate multiple time points to understand the lifecycle of the nanoparticle biocorona as its content could change over time [27]. Future study is needed examining the lifecycle of the nanoparticle-biocorona by assessing biocoronal content over various time courses. It is also likely that the nanoparticle biocorona that exists in the extracellular physiological media will not be identical to the biocorona intracellularly. An assessment of the biocorona that forms on silver nanoparticles by hyperspectral darkfield microscopy demonstrated that the spectral profile of the nanoparticle-biocorona was different extracellularly compared to intracellularly [17]. This suggests surface modifications to the nanoparticle following internalization and likely alterations in the biocorona. Through their magnetic properties, it has been possible to extricate internalized silica-coated iron oxide nanoparticles from cells [28]. These internalized iron oxide nanoparticles retained a significant portion of the original hard corona while being trafficked through cells [28]. This finding suggests limited intracellular modifications to the iron oxide nanoparticle-biocorona. Knowledge gained from evaluating the components of the intracellular biocorona could provide details into specific cell signaling pathways that the nanoparticle interacts and interferes with following internalization. This intracellular biocorona is likely related to the subcellular localization of nanoparticles following internalization. Nanoparticles in different subcellular compartments will interact with unique biomolecules resulting in variable responses. Studies have evaluated the extracellular biocorona, whereas the intracellular nanoparticle-biocorona has received limited investigation but may be responsible for many cellular responses.

The extracellular biocorona may not only influence uptake of the nanoparticle by cells but also the subsequent subcellular location resulting in differential cellular responses. Cellular compartments where nanoparticles 
are known to localize internally with lower $\mathrm{pH}$ such as lysosomes may result in the release of the biocoronal components and/or nanoparticles resulting in toxicological responses [29,30]. These released biocoronal components due to their interactions with the nanoparticle surface likely have undergone a conformational modification resulting in an endoplasmic reticulum stress response. Recent study has demonstrated that silver nanoparticles can induce an endoplasmic reticulum response; however, it is unknown if this is due to the release of conformationally modified extracellular biomolecules from the surface of the nanoparticles, nanoparticles inducing conformational changes in biomolecules intracellularly, or direct nanoparticle interactions with the endoplasmic reticulum $[31,32]$. In the case of silica, surface curvature has been demonstrated to mediate the nanoparticle-protein interaction surface resulting in alterations in the protein's secondary structure [33]. Specifically, the larger the diameter of the nanoparticle, the more nanoparticle-protein interactive surface is available resulting in increased conformational changes. The ability of the nanoparticle-biocorona to possibly induce changes in subcellular localization and conformational changes in protein structure, as well as an endoplasmic reticulum stress response requires further assessment as changes in these may result in unintended cellular responses. It is likely that targeted nanoparticles that result in structural alterations of specific proteins could be the basis for numerous therapeutic strategies.

One of the primary limitations of the nanoparticle-biocorona field has been the qualitative nature of much of the research. This is mostly due to the field being in its early stages and the need for the development of study designs and technologies that are more quantitative. Simple model systems utilizing single proteins have been used to examine the dynamics of the biocorona. Specifically, these models have investigated the kinetics of individual protein-nanoparticle interactions forming the biocorona through the evaluation of association and dissociation rates, affinities, and stoichiometries [3, 34, 35]. These studies demonstrated that the kinetics of interaction were dependent on a variety of parameters including time, particle characteristics (surface curvature, composition, and coating), and the identity of the individual protein being evaluated. Specifically, Cedervall et al. demonstrated that albumin and fibrinogen associated and dissociated at higher rates than apolipoprotein A-I and most other proteins from the plasma [34]. Although demonstrating slower kinetics, apolipoprotein A-I had a high affinity for $\mathrm{N}$-isopropylacrylamide-co-N-tert-butylacrylamide copolymer nanoparticles. This means that although highly abundant proteins in the circulation may rapidly interact with the nanoparticles surface, over time the nanoparticle will become coated with more low abundant proteins that have higher affinities for the surface. This kinetics-based research approach further demonstrates the challenges of the nanoparticle-biocorona as multiple factors need to be taken into consideration. Future research is needed to understand the highly complex kinetics of nanoparticlebiomolecular interactions that take place on the surface of nanoparticles as biomolecules compete for space. These kinetic relationships likely are modified by a variety of parameters including time, nanoparticle characteristics, protein characteristics, biological compartment, and human individuality of the biological milieu.

\subsection{Physiological-related differences in the biocorona}

The nanoparticle-biocorona is also not uniform between different physiological environments. Therefore, human routes of exposure need to be taken into account when evaluating the nanoparticle-biocorona. For example, an inhaled nanoparticle will absorb a different biocorona compared to one that was injected or ingested and may result in profoundly different cellular responses. To date much of the evaluation of the nanoparticle-biocorona has focused on the route of injection and has utilized serum or plasma. Proteomic evaluation, however, has identified that unique biocoronas form on the surface of nanoparticles following inhalation that are rich in surfactant protein A, napsin A, and complement proteins [36]. The identity of these proteins that form the biocorona in the respiratory tract lining fluid suggests opsonization of the nanoparticles allowing for a pulmonary immune response. Exposure evaluating the role of the biocorona in nanoparticle dermal exposures demonstrated differences in nanoparticle uptake due to addition of an IgGbiocorona [37]. Specifically, the addition of IgG to the surface of $20 \mathrm{~nm}$ silica silver nanoparticles enhanced uptake, whereas the uptake of other nanoparticles was either unchanged or reduced following addition of the IgG to their surface. To date the biocorona that forms due to routes of nanoparticle exposure other than intravenous such as inhalation, ingestion, and dermal are understudied in comparison. These other routes of exposure require further evaluation as human exposures continue to rise due to their increased commercialization, introduction into our environments, and biomedical applications. Specifically, inhaled, ingested, or dermally applied nanotherapeutics will have to consider these unique biocorona that form on the surface of nanoparticles. 
Laboratory evaluation is necessary for evaluation of the nanoparticle-biocorona; however, translating laboratory findings to humans may present distinct challenges. Preforming the biocorona is often done prior to their introduction into in vitro exposure systems. This is done in order to remove the complexity of the highly dynamic soft corona thus allowing for the study of the more stable hard corona. It is likely that the hard biocorona that is associated with nanoparticles acellularly is very similar to the hard biocorona that forms when nanoparticles are introduced into cell culture environments and macromolecules are present since it forms rather rapidly. There may be significant differences between these preformed biocoronas and those that form naturally on nanoparticles in vivo. This is due to many variables including changes in the nanoparticle's agglomerative state, the presence of flow conditions, the passage of the nanoparticle through different biological compartments, and others. Specifically, it has been shown that liposomes injected into a mouse model and later collected acquire a uniquely complex biocorona compared to in vitro incubation in mouse plasma [38]. Further translation between the biocoronas that form in rodent models and humans may be difficult due to differences in macromolecule content and physiological differences. Sahneh et al. demonstrated through pharmacokinetic modeling that, due to increased circulation times, nanoparticles would be present in the bloodstream of humans for longer periods of time than in rodents prior to reaching their targets [27]. This increased time would allow for a different biocorona to form in a human compared to in a rodent and would likely influence biodistribution. This highlights the need for future study evaluating differences in the biocorona due to variability between species, physiology, and in vitro and in vivo systems. Further, these studies suggest that certain animal models may be more appropriate for translating nanoparticle research to humans. Specifically, physiologically based pharmacokinetic modeling of gold nanoparticles has suggested that rats or pigs are more translatable models than typically utilized mouse models [39].

Underlying disease states are also known to modify the physiological environment and may result in alterations in the biocorona due to disease-related differences in available biomolecules. A recent evaluation of how underlying disease states modify the biocorona that forms on iron oxide nanoparticles utilized serum from healthy and hyperlipidemic rats [8]. Incubation in these different serums was determined to result in the formation of distinct biocoronas. There was less abundance of common proteins on the surface of nanoparticles incubated in the hyperlipidemic serum. Further incubation in the hyperlipidemic serum resulted in increased cholesterol content of the biocorona compared to normal serum. It is possible that the increased cholesterol inhibited the association of proteins. These different disease-state biocoronas were also found to modify cellular responses. Specifically, the hyperlipidemic biocorona was determined to induce a greater inflammatory response in endothelial cells compared with iron oxide nanoparticles with a healthy biocorona. This study demonstrates the importance of disease-related differences in the biocorona and its toxicological implications. As diseases such as hyperlipidemia continue to expand in our population, nanoparticles for biomedical applications may need to be evaluated in models systems that mimic these prominent disease environments as opposed to the healthy environments commonly used in laboratory settings. Individuals with underlying disease will likely form a unique biocorona on nanoparticles compared to healthy individuals leading to unforeseen biological responses. It may be necessary based on the route of administration for nanoparticles to be screened in these different disease environments. Many diseases also have progressive modifications physiologically such as kidney disease. This may mean that an individual at different stages of disease progression will form different biocoronas on nanoparticles throughout their disease progression, possibly altering nanoparticle functionality and biological responses. These disease-related differences in the formation of the biocorona need to be taken into account while prescribing nanotherapeutics as they may result in altered responses to the nanoparticles such as inhibited therapeutic efficiency, clearance, and/ or toxicity. These studies taking into account underlying disease states need to be prioritized based on prominent human diseases as well as the disease-state of the human in which the nanoparticle will be utilized.

\subsection{Nanoparticle functionality and therapeutically useful modifications of the biocorona}

Addition of the nanoparticle-biocorona has been shown to decrease the functionality of nanoparticles often by interfering with targeting of nanotherapeutics. Specifically, addition of the biocorona has been evaluated for its role in altering the effectiveness of iron oxide nanoparticles as MRI contrast agents. Research has demonstrated that addition of the biocorona on superparamagnetic iron oxide nanoparticles does not significantly change T2 
relaxation times; however, it can alter binding to cells [40, 41]. Further, the addition of the biocorona has been demonstrated to inhibit the targeting capabilities of transferrin-coated nanoparticles thus reducing their functionality [42]. The addition of surfactants to the surface of nanoparticles has been used to decrease protein association thus allowing for reduction in the clearance of nanoparticles by the immune system [43]. Nevertheless, this also inhibits interactions with targeted cell populations reducing the functionality of the nanoparticles. Recently, however, it has been demonstrated that backfilling the surface of a targeted nanoparticle with polyethylene glycol can reduce the association of serum proteins allowing for less biocorona-related inhibition of targeting [44]. Biomedical applications that utilized nanotechnology must consider the influence of the biocorona during product synthesis or the benefits of nanotechnology may be lost.

Manipulation of the nanoparticle-biocorona may also be beneficial for the biomedical application of nanoparticles. It is likely that nanoparticles could be formulated to associate biocoronas rich in specific biomolecules thus assisting in nanotherapeutic retention and targeting. Data accessing the identity of the biocorona on the surface of nanoparticles is necessary; however, being able to manipulate the biocorona may be needed for a variety of applications. A recent study has determined that surfactant titration allows for the selective removal of apolipoprotein AI from the hard biocorona that forms on the surface of $100 \mathrm{~nm}$ silicon oxide nanoparticles following incubation in human plasma [45]. This study demonstrates that individual components of the biocorona can possibly be controlled allowing for the formation of specific nanoparticle-biocoronas that can be tuned for individual purposes. Further study, however, needs to be performed to produce protocols allowing for the selective removal of other biocoronal components allowing for the synthesis of specific biocoronas. It is also possible to utilize nanoparticles that preferentially bind certain biomolecules such as cholesterol in order to assist in the clearance of the biomolecule from the body. These nanoparticles that take advantage of the natural binding of biomolecules for their removal from the body may prove to be useful treatments for a variety of diseases in the future. Lastly, nanoparticles may be synthesized to preferentially associate with a specific biocorona once injected, allowing for the enhancement of nanoparticle targeting. Nanoparticles which preferentially bind large amounts of apolipoproteins have shown an ability to more efficiently pass through the blood brain barrier [46-48]. Instead of seeing the biocorona as a challenge to overcome, many possible therapeutic applications may be able to take advantage of the nanoparticle-biocorona and use it as a natural surface coating to increase their usefulness.

\subsection{Biocorona-induced alterations in dosimetry}

Biomedical applications that utilize nanotechnology are often screened using cell culture models. This often involves growing adherent cells at the bottom of a plate with media on top and injecting nanoparticles into the media. The nanoparticles will eventually settle and interact with the cells allowing for testing of various parameters. Due to their small size and high surface area, nanoparticle dosimetry is difficult and could easily be influenced by differential biocoronas. The addition of unique biocoronas on the surface of the nanoparticles thereby increasing or decreasing the size of the nanoparticle agglomerates may result in distinctive settling velocities causing alterations in dosimetry. These differences in dosimetry make comparisons of effects between nanoparticles challenging as each set of cells interacted with differing amounts of nanoparticles. Use of dosimetry models, such as the in vitro sedimentation, diffusion, and dosimetry model; computational fluid dynamics model; the distorted grid model; and others which predict delivered nanoparticle doses in in vitro scenarios could be beneficial in nullifying this challenge [49-52]. The use of these models would allow for comparable dosing in cell culture models of not only nanoparticles but nanoparticles with various biocoronas. The information gained is needed for cell culture experimentation to set doses based on the number of nanoparticles that cells interact with over a period of time. These studies are a challenge, however, due to the diversity of nanoparticles and the complexity of the biocorona. Dosimetry differences related to the biocorona, however, need to be taken into consideration prior to experimentation for the generation of more relevant and reliable data in the early screening of nanoparticles.

\subsection{Investigation of the secondary or soft biocorona}

To date the majority of research has evaluated the hard corona that forms on nanoparticles. This hard corona is the initial layer of biomolecules which is relatively stable and is governed by nanoparticle-biomolecule interactions making it easier to evaluate. The soft corona or the secondary corona, which is composed of biomolecules 
interacting with the hard corona, is less studied due to its more dynamic nature. There are multiple challenges associated with studying the soft corona. The primary issue is how to distinguish it from the hard corona when isolating biomolecules from the surface of the nanoparticle. Further, any assessment is a snapshot due to its dynamic nature. The secondary biocorona, however, is likely of importance because it continually may produce modified biomolecules as they associate and disassociate. Further, this secondary biocorona may also govern many biocorona-cell interactions, and as the secondary corona undergoes alterations in its content, these interactions may also change. A recent study formed a hard corona on the surface of hydroxyethyl starch nanocapsules and then incubated them with individual proteins [53]. The association of the secondary biocorona was then evaluated through the use of isothermal titration calorimetry and dynamic light scattering. This study was able to determine the association of these proteins with the hard corona forming the secondary biocorona on nanoparticles. Ultimately, this study demonstrates that the assessment of the secondary biocorona is possible; however, future studies with similar novel approaches and advances are needed. Due to the dynamic nature of the secondary biocorona, its assessment will likely require the involvement of computational modelers to predict the protein-protein interactions that govern the secondary biocorona and its changes over time. This type of data will allow for the early assessment of the nanoparticle-secondary biocorona prior to biological experimentation, thus making the overall process more cost effective.

\subsection{Alterations in dissolution}

Many nanoparticles such as silver nanoparticles undergo dissolution; this release of ions is related to their antimicrobial activity. Further alterations in silver nanoparticle dissolution due to modifications in their physicochemical properties have been shown to influence toxicological outcomes in vitro [54]. The addition of the biocorona may alter this dissolution resulting in the stabilization or destabilization of the nanoparticle structure. For instance, biomolecules that strongly associate may reduce the dissociation of ions from the surface of nanoparticles, whereas weak associations may result in increased dissociation of ions due to continual interactions. Investigation of single-protein biocoronas demonstrated that albumin stabilized silver nanoparticles reducing dissolution, whereas high-density lipoprotein increased dissolution compared to when no biocorona was present $[9,22]$. It is likely that the addition of highdensity lipoprotein removed citrate groups which were used as a surface coating for these silver nanoparticles thereby destabilizing the silver nanoparticles and resulting in dissolution. Further disruption of the bovine serum albumin coating on silver nanoparticles has been shown to increase dissolution supporting the stabilization of silver nanoparticles via specific biocoronal proteins [55]. This ability of the biocorona to stabilize or destabilize the nanoparticle's structure may alter the nanoparticle's functionality, toxicity, and biopersistence. Further, the impact of the biocorona on stability/dissolution of metal oxide nanoparticles is one of the most direct demonstrations of a functional role of the biocorona in regards to toxicological impact.

\section{Conclusions}

The nanoparticle-biocorona presents a unique challenge for the safe and effective biomedical application of nanoparticles. The biocorona imparts a distinct identity to the nanoparticle influencing its characteristics, functionality, and toxicity. To understand how nanoparticles can be utilized in biological applications, the biocorona must be taken into account as this is the first biological interaction that occurs following introduction into the body and governs all subsequent cellular interactions. Currently, research has begun to examine its potential impact on the uses of nanotechnology and has raised enough concern to warrant further investigation. Specific areas of further investigation include (1) further correlations between nanoparticle properties and biomolecule association, including non-protein components of the biocorona, (2) lifecycle assessment of the nanoparticle-biocorona, (3) physiological impact on biocorona formation, (4) biocorona modifications to nanoparticle functionality, (5) secondary biocorona formation on nanoparticles and its biological impact, (6) biocorona-induced alterations in dosimetry and biodistribution, and (7) manipulation of the nanoparticle-biocorona for therapeutic and diagnostic applications.

Acknowledgments: This work was supported by the NIEHS grant K99 ES024392 (JHS). Any opinions, findings, conclusions, or recommendations expressed herein are those of the authors and do not necessarily reflect the views of the National Institute of Environmental Health Sciences/NIH. 


\section{References}

[1] Walkey CD, Chan WC. Understanding and controlling the interaction of nanomaterials with proteins in a physiological environment. Chem. Soc. Rev. 2012, 41, 2780-2799.

[2] O’Connell DJ, Baldelli Bombelli F, Pitek AS, Monopoli MP, Cahill DJ, Dawson KA. Characterization of the bionano interface and mapping extrinsic interactions of the corona of nanomaterials. Nanoscale 2015, 7, 15268-15276.

[3] Podila R, Chen R, Ke PC, Brown JM, Rao AM. Effects of surface functional groups on the formation of nanoparticle-protein corona. Appl. Phys. Lett. 2012, 101, 263701.

[4] Sengupta B, Gregory WE, Zhu J, Dasetty S, Karakaya M, Brown JM, Rao AM, Barrows JK, Sarupria S, Podila R. Influence of carbon nanomaterial defects on the formation of protein corona. RSC Adv. 2015. 5, 82395-82402.

[5] Shannahan JH, Lai X, Ke PC, Podila R, Brown JM, Witzmann FA. Silver nanoparticle protein corona composition in cell culture media. PLoS One 2013, 8, e74001.

[6] Saha K, Rahimi M, Yazdani M, Kim ST, Moyano DF, Hou S, Das R, Mout R, Rezaee F, Mahmoudi M, Rotello VM. Regulation of macrophage recognition through the interplay of nanoparticle surface functionality and protein corona. ACS Nano. 2016, 10, 4421-4430.

[7] Lesniak A, Salvati A, Santos-Martinez MJ, Radomski MW, Dawson KA, Åberg C. Nanoparticle adhesion to the cell membrane and its effect on nanoparticle uptake efficiency. J. Am. Chem. Soc. 2013, 135, 1438-1444.

[8] Shannahan JH, Fritz KS, Raghavendra AJ, Podila R, Persaud I, Brown JM. Disease-induced disparities in formation of the nanoparticle-biocorona and the toxicological consequences. Toxicol. Sci. 2016, 406-416.

[9] Duran N, Silveira CP, Durán M, Martinez DS. Silver nanoparticle protein corona and toxicity: a mini-review. J. Nanobiotechnol. 2015, 13, 55.

[10] Neagu M, Piperigkou Z, Karamanou K, Engin AB, Docea AO, Constantin C, Negrei C, Nikitovic D, Tsatsakis A. Protein biocorona: critical issue in immune nanotoxicology. Arch. Toxicol. 2016. [Epub ahead of print].

[11] Monopoli MP, Aberg C, Salvati A, Dawson KA. Biomolecular coronas provide the biological identity of nanosized materials. Nat. Nanotechnol. 2012, 7, 779-786.

[12] Mahon E, Salvati A, Baldelli Bombelli F, Lynch I, Dawson KA. Designing the nanoparticle-biomolecule interface for "targeting and therapeutic delivery". J. Control. Release 2012, 161, 164-174.

[13] Caracciolo G, Farokhzad OC, Mahmoudi M. Biological identity of nanoparticles in vivo: clinical implications of the protein corona. Trends Biotechnol. 2016. [Epub ahead of print].

[14] Monopoli MP, Pitek AS, Lynch I, Dawson KA. Formation and characterization of the nanoparticle-protein corona. Methods Mol. Biol. 2013, 1025, 137-155.

[15] Tenzer S, Docter D, Rosfa S, Wlodarski A, Kuharev J, Rekik A, Knauer SK, Bantz C, Nawroth T, Bier C, Sirirattanapan J, Mann W, Treuel L, Zellner R, Maskos M, Schild H, Stauber RH. Nanoparticle size is a critical physicochemical determinant of the human blood plasma corona: a comprehensive quantitative proteomic analysis. ACS Nano. 2011, 5, 7155-7167.
[16] Shannahan JH, Brown JM, Chen R, Ke PC, Lai X, Mitra S, Witzmann FA. Comparison of nanotube-protein corona composition in cell culture media. Small 2013, 9, 2171-2181.

[17] Shannahan JH, Podila R, Brown JM. A hyperspectral and toxicological analysis of protein corona impact on silver nanoparticle properties, intracellular modifications, and macrophage activation. Int. J. Nanomed. 2015, 10, 6509-6521.

[18] Walkey CD, Olsen JB, Song F, Liu R, Guo H, Olsen DW, Cohen Y, Emili A, Chan WC. Protein corona fingerprinting predicts the cellular interaction of gold and silver nanoparticles. ACS Nano. 2014, 8, 2439-2455.

[19] Kelly PM, Åberg C, Polo E, O'Connell A, Cookman J, Fallon J, Krpetić Ž, Dawson KA. Mapping protein binding sites on the biomolecular corona of nanoparticles. Nat. Nanotechnol. 2015, 10, 472-479.

[20] Shannahan JH, Sowrirajan H, Persaud I, Podila R, Brown JM. Impact of silver and iron nanoparticle exposure on cholesterol uptake by macrophages. J. Nanomater. 2015, 2015, 1-12.

[21] Shannahan JH, Bai W, Brown JM. Implications of scavenger receptors in the safe development of nanotherapeutics. Receptors Clin. Investig. 2015, 2, e811.

[22] Shannahan JH, Podila R, Aldossari AA, Emerson H, Powell BA, Ke PC, Rao AM, Brown JM. Formation of a protein corona on silver nanoparticles mediates cellular toxicity via scavenger receptors. Toxicol. Sci. 2015, 143, 136-146.

[23] Zani IA, Stephen SL, Mughal NA, Russell D, Homer-Vanniasinkam S, Wheatcroft SB, Ponnambalam S. Scavenger receptor structure and function in health and disease. Cells 2015, 4, 178-201.

[24] Pozzi D, Colapicchioni V, Caracciolo G, Piovesana S, Capriotti AL, Palchetti S, De Grossi S, Riccioli A, Amenitsch H, Laganà A. Effect of polyethyleneglycol (PEG) chain length on the bio-nano-interactions between PEGylated lipid nanoparticles and biological fluids: from nanostructure to uptake in cancer cells. Nanoscale 2014, 6, 2782-2792.

[25] Lundqvist M, Stigler J, Elia G, Lynch I, Cedervall T, Dawson KA. Nanoparticle size and surface properties determine the protein corona with possible implications for biological impacts. Proc. Natl. Acad. Sci. U. S. A. 2008, 105, 14265-14270.

[26] Agarwal R, Roy K. Intracellular delivery of polymeric nanocarriers: a matter of size, shape, charge, elasticity and surface composition. Ther. Deliv. 2013, 4, 705-723.

[27] Sahneh FD, Scoglio CM, Monteiro-Riviere NA, Riviere JE. Predicting the impact of biocorona formation kinetics on interspecies extrapolations of nanoparticle biodistribution modeling. Nanomedicine (Lond) 2015, 10, 25-33.

[28] Bertoli F, Davies GL, Monopoli MP, Moloney M, Gun'ko YK, Salvati A, Dawson KA. Magnetic nanoparticles to recover cellular organelles and study the time resolved nanoparticlecell interactome throughout uptake. Small 2014, 10, 3307-3315.

[29] Mishra AR, Zheng J, Tang X, Goering PL. Silver nanoparticleinduced autophagic-lysosomal disruption and NLRP3-inflammasome activation in HepG2 cells is size-dependent. Toxicol. Sci. 2016, 150, 473-487.

[30] Wang F, Yu L, Monopoli MP, Sandin P, Mahon E, Salvati A, Dawson KA. The biomolecular corona is retained during nanoparticle uptake and protects the cells from the damage induced by cationic nanoparticles until degraded in the lysosomes. Nanomedicine 2013, 9, 1159-1168. 
[31] Christen V, Capelle M, Fent K. Silver nanoparticles induce endoplasmatic reticulum stress response in zebrafish. Toxicol. Appl. Pharmacol. 2013, 272, 519-528.

[32] Zhang R, Piao MJ, Kim KC, Kim AD, Choi JY, Choi J, Hyun JW. Endoplasmic reticulum stress signaling is involved in silver nanoparticles-induced apoptosis. Int. J. Biochem. Cell. Biol. 2012, 44, 224-232.

[33] Lundqvist M, Sethson I, Jonsson BH. Protein adsorption onto silica nanoparticles: conformational changes depend on the particles' curvature and the protein stability. Langmuir 2004 , 20, 10639-10647.

[34] Cedervall T, Lynch I, Lindman S, Berggård T, Thulin E, Nilsson H, Dawson KA, Linse $S$. Understanding the nanoparticle-protein corona using methods to quantify exchange rates and affinities of proteins for nanoparticles. Proc. Natl. Acad. Sci. USA. 2007, 104, 2050-2055.

[35] Milani S, Bombelli FB, Pitek AS, Dawson KA, Rädler J. Reversible versus irreversible binding of transferrin to polystyrene nanoparticles: soft and hard corona. ACS Nano. 2012, 6, 2532-2541.

[36] Kumar A, Bicer EM, Morgan AB, Pfeffer PE, Monopoli M, Dawson KA, Eriksson J, Edwards K, Lynham S, Arno M, Behndig AF, Blomberg A, Somers G, Hassall D, Dailey LA, Forbes B, Mudway IS. Enrichment of immunoregulatory proteins in the biomolecular corona of nanoparticles within human respiratory tract lining fluid. Nanomedicine 2016, 12, 1033-1043.

[37] Monteiro-Riviere NA, Samberg ME, Oldenburg SJ, Riviere JE. Protein binding modulates the cellular uptake of silver nanoparticles into human cells: implications for in vitro to in vivo extrapolations? Toxicol. Lett. 2013, 220, 286-293.

[38] Hadjidemetriou M, Al-Ahmady Z, Mazza M, Collins RF, Dawson $\mathrm{K}$, Kostarelos K. In vivo biomolecule corona around bloodcirculating, clinically used and antibody-targeted lipid bilayer nanoscale vesicles. ACS Nano. 2015, 9, 8142-8156.

[39] Lin Z, Monteiro-Riviere NA, Kannan R, Riviere JE. A computational framework for interspecies pharmacokinetics, exposure and toxicity assessment of gold nanoparticles. Nanomedicine (Lond) 2016, 11, 107-119.

[40] Hill A, Payne CK. Impact of serum proteins on MRI contrast agents: cellular binding and T2 relaxation. RSC Adv. 2014, 4, 31735-31744.

[41] Yallapu MM, Chauhan N, Othman SF, Khalilzad-Sharghi V, Ebeling MC, Khan S, Jaggi M, Chauhan SC. Implications of protein corona on physico-chemical and biological properties of magnetic nanoparticles. Biomaterials 2015, 46, 1-12.

[42] Salvati A, Pitek AS, Monopoli MP, Prapainop K, Bombelli FB, Hristov DR, Kelly PM, Åberg C, Mahon E, Dawson KA. Transferrin-functionalized nanoparticles lose their targeting capabilities when a biomolecule corona adsorbs on the surface. Nat. Nanotechnol. 2013, 8, 137-143.

[43] Sanchez-Moreno P, Buzón P, Boulaiz H, Peula-García JM, Ortega-Vinuesa JL, Luque I, Salvati A, Marchal JA. Balancing the effect of corona on therapeutic efficacy and macrophage uptake of lipid nanocapsules. Biomaterials 2015, 61, 266-278.

[44] Dai Q, Walkey C, Chan WC. Polyethylene glycol backfilling mitigates the negative impact of the protein corona on nanoparticle cell targeting. Angew. Chem. Int. Ed. Engl. 2014, 53, 5093-5096.

[45] Maiolo D, Bergese P, Mahon E, Dawson KA, Monopoli MP. Surfactant titration of nanoparticle-protein corona. Anal. Chem. $2014,86,12055-12063$.
[46] Kreuter J. Mechanism of polymeric nanoparticle-based drug transport across the blood-brain barrier (BBB). J. Microencapsul. 2013, 30, 49-54.

[47] Neves AR, Queiroz JF, Reis S. Brain-targeted delivery of resveratrol using solid lipid nanoparticles functionalized with apolipoprotein E. J. Nanobiotechnol. 2016, 14, 27.

[48] Neves AR, Queiroz JF, Weksler B, Romero IA, Couraud PO, Reis S. Solid lipid nanoparticles as a vehicle for brain-targeted drug delivery: two new strategies of functionalization with apolipoprotein E. Nanotechnology 2015, 26, 495103.

[49] Hinderliter PM, Minard KR, Orr G, Chrisler WB, Thrall BD, Pounds JG, Teeguarden JG. ISDD: a computational model of particle sedimentation, diffusion and target cell dosimetry for in vitro toxicity studies. Part Fibre Toxicol. 2010, 7, 36.

[50] Cohen JM, DeLoid GM, Demokritou P. A critical review of in vitro dosimetry for engineered nanomaterials. Nanomedicine (Lond) 2015, 3015-3032.

[51] Liu R, Liu HH, Ji Z, Chang CH, Xia T, Nel AE, Cohen Y. Evaluation of toxicity ranking for metal oxide nanoparticles via an in vitro dosimetry model. ACS Nano. 2015, 9, 9303-9313.

[52] DeLoid GM, Cohen JM, Pyrgiotakis G, Pirela SV, Pal A, Liu J, Srebric J, Demokritou P. Advanced computational modeling for in vitro nanomaterial dosimetry. Part Fibre Toxicol. 2015, 12, 32.

[53] Winzen S, Schoettler S, Baier G, Rosenauer C, Mailaender V, Landfester K, Mohr K. Complementary analysis of the hard and soft protein corona: sample preparation critically effects corona composition. Nanoscale 2015, 7, 2992-3001.

[54] Munusamy P, Wang C, Engelhard MH, Baer DR, Smith JN, Liu C, Kodali V, Thrall BD, Chen S, Porter AE, Ryan MP. Comparison of $20 \mathrm{~nm}$ silver nanoparticles synthesized with and without a gold core: structure, dissolution in cell culture media, and biological impact on macrophages. Biointerphases 2015, 10, 031003.

[55] Martin MN, Allen AJ, MacCuspie RI, Hackley VA. Dissolution, agglomerate morphology, and stability limits of protein-coated silver nanoparticles. Langmuir 2014, 30, 11442-11452.

\section{Bionote}

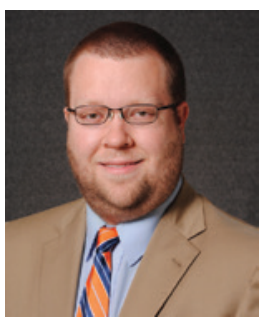

Jonathan Shannahan

School of Health Sciences, Purdue

University, 550 Stadium Mall Dr. 47907,

West Lafayette, Indiana, USA,

Tel.: +765-494-2326,

jshannah@purdue.edu

Jonathan Shannahan is an Assistant Professor of Health Sciences and Toxicology in the School of Health Sciences at Purdue University. He studied toxicology at the University of North Carolina Chapel Hill and received additional training in nanotoxicology at the University of Colorado - Anschutz Medical Campus. He has published numerous peer-reviewed scientific articles on air pollution health effects, susceptibility, nanotoxicology, and the nanoparticle-biocorona. His current research interests focus on the influence of underlying disease states on nanoparticle functionality and toxicity. 